\title{
Objectively Measured Association Between Air Pollution and Physical Activity and Sedentary Behavior Among College Students in Beijing
}

\section{Miao Yu}

Renmin University of China

Yin Wu

Tsinghua University

Shelby Paige Gordon

Columbia University

Jiali Cheng

Tsinghua University

\section{Panpan Chen}

Tsinghua University

\section{Yangyang Wang}

Tsinghua University

Hongjun Yu ( $\square$ yuhj12@mail.tsinghua.edu.cn )

Tsinghua University https://orcid.org/0000-0003-1687-934X

\section{Research}

Keywords: Air pollution, AQI, PM2.5, Physical activity, Sedentary behavior, Youth

Posted Date: June 17th, 2020

DOl: https://doi.org/10.21203/rs.3.rs-33667/v1

License: (ㅇ) (i) This work is licensed under a Creative Commons Attribution 4.0 International License. Read Full License

Version of Record: A version of this preprint was published at Environmental Research on March 1st, 2021. See the published version at https://doi.org/10.1016/j.envres.2020.110492. 


\section{Abstract}

Background: Air pollution has become a major environmental health risk factor, notably in China. Air pollution potentially has the impact of human populations' health behavior. Gaps in scientific literature remain regarding more accurately estimates the relationship between air pollution and sedentary behavior in China. The purpose of this study is to examine the association between hourly air pollution AP on hourly physical activity (PA) and sedentary behavior (SB) among college students in Beijing, China. The secondary aim was to examine such associations varied at specific times.

Methods: A total of 340 participants were recruited from the Tsinghua University, in Beijing, China. Accelerometers provided PA measures, including moderate-to-vigorous physical activity (MVPA), walking steps, energy expenditure and sedentary time for 7 consecutive days. Corresponding AP data by the Beijing Municipal Ecological Environment Bureau in the closed site (Wan Liu site) at Tsinghua University were collected to include average hourly air quality index (AQI) and $\mathrm{PM}_{2.5}\left(\mathrm{\mu g} / \mathrm{m}^{3}\right)$. Associations were estimated using linear individual fixed-effect regressions.

Results: A one level increase in hourly air quality index (AQI) was associated with a reduction in one-hour MVPA by 0.083 minutes $(95 \% \mathrm{Cl}=[-0.137,-0.029]), 8.8$ walking steps $(95 \% \mathrm{Cl}=[-15.0,-2.6])$, and $0.65 \mathrm{kcals}$ of energy expenditure $(95 \% \mathrm{Cl}=-[1.03,-0.27]) . \mathrm{A}$ $10 \mu \mathrm{g} / \mathrm{m}^{3}$ increase in AP concentration in hourly $\mathrm{PM}_{2.5}$ was associated with a reduction in one-hour MVPA by 0.021 minutes $(95 \% \mathrm{Cl}=$ $[-0.033,-0.010]), 2.2$ walking steps $(95 \% \mathrm{Cl}=[-3.5,-0.9]), 0.170$ kcals of energy expenditure $(95 \% \mathrm{Cl}=[-0.250,-0.089])$, and an increase in one-hour $\mathrm{SB}$ by $0.045(95 \% \mathrm{Cl}=[0.005,0.0845])$. At a specific time, there are stronger negative associations of $A Q I$ and $P M_{2.5}$ to $P A$ at $8 \mathrm{am}, 4 \mathrm{pm}, 5 \mathrm{pm}$ and $7 \mathrm{pm}$. Similarly, stronger positive associations were found at one hour AQI and PM 2.5 with SB at 8 am, 9 am, $11 \mathrm{am}$, and $7 \mathrm{pm}$.

Conclusions: AP may discourage PA and increases SB among freshman students living in Beijing, China. The impact of AP on PA and sedentary behavior at a specific time may be different.

\section{Introduction}

Air pollution (AP) has become a major environmental health risk factor for overall health worldwide. AP levels in China have increased rapidly due to the industrialization use of fossil fuel and population growth [1]. Previous evidences has showed that exposure to AP has been detrimental to various health outcomes e.g., cardiovascular disease, stroke, lung cancer, respiratory disease, all-cause mortality, sleep apnea, and depression [2-10].

Strong evidence indicates that engaging in regular physical activity (PA) has many health benefits including increased effective weight management, reduced risk of all-cause mortality, and prevention and management of chronic diseases, e.g., cardiovascular diseases, diabetes, colon and breast cancer, hypertension, coronary heart disease, and osteoarthritis [11-14]. While there are several health benefits to PA, performing PA under high levels of AP may accelerate the risks for adverse health effects such as asthma attacks and heart or lung pathologies [15-17]. Outdoor parks and playgrounds are common places to perform PA in China [18], and the presence of AP in China may further discourage young adults from engaging in regular PA and exercise [19].

Independent of PA, prolonged sedentary behavior (SB) has found to lead to negative health outcomes, including increased risk of obesity, type 2 diabetes, cardiovascular diseases and all-cause mortality among adolescents and adults [20-25].

Substantial research has measured the effects of AP on health outcomes, but few studies have determined its impact on healthrelated behaviors, specifically PA and SB behavior. There are three major gaps in the scientific literature that need for further investigation. First, existing research on the associations between AP, PA and SB are limited. Second, most previous studies have used a self-reported survey method [26], therefore, existing studies were subject to social desirability bias and limited by the frequency of PA performance and SB data (i.e., week by week, month by month, or year by year). This study is the first to provide precise, objectively measured data through use of the digital accelerometers to measure PA and SB among university freshman students in China. A digital accelerometer omits the chance for social desirability bias while providing minute-by-minute measurements of PA and SB. Third, to date, all previous studies estimated the impact of AP on health-related behaviors week on a week, month by month, or year by year basis. There is currently no study which investigates the relationship between AP and PA and SB at a specific time. 
This study reported the associations between AP, PA and SB among university freshman students living in Beijing, China. Objectively measured data were collected by using of the digital accelerometers during this study. The final sample size was 340 participants. Hourly AP data and minute-by-minute PA and SB data were measured. We hypothesized that in response to high levels of AP, university freshmen reduced their PA behaviors and increased their SB. We also hypothesized that such associations varied at a specific time.

\section{Materials And Methods}

\subsection{Participants and Sampling Procedure}

The study was conducted from the period of November 2017 to April 2018. All freshmen students are required to take the same physical education class in Tsinghua University. 344 freshmen students who were enrolled in the required physical education class were recruited as participants. Participants were recruited 6 times during the study period (Nov 6-14, 2017; Nov 15-30, 2017; Dec 18, 2017-Jan 5, 2018; Feb 27- Mar 6, 2018; Mar 12-Mar 19, 2018; and Mar 26-APr 3, 2018). Upon acceptance, the subjects were asked to visit our lab to get a wGT3X-BT device fitted. Height and weight were measured to the nearest $0.1 \mathrm{~kg}$ using a Seca beam scale (Tsinghua Tongfang, S5000, China). Each participant completed one paper-pencil based health survey on demographic status (age, gender, ethnicity, lifestyle smoking, drinking, and mental and physical health conditions). Of the 344 recruited, 340 subjects had completed the study (4 participants' data were excluded due to 2 devices errors, and 2 missing accelerometers that were not returned). All participants gave informed consent, and the study was approved by the Tsinghua University Institutional Review Board (IRB \#2017DX02_11).

\subsection{Measures}

\subsubsection{PA and SB measurement}

The participants were instructed to wear a wGT3X-BT accelerometer over the right hip on a waist band for at least 10 hours a day (excluding sleep time) over 7 consecutive days. The participants removed the device it only during showers, bathing, swimming or other water activities. The recording epoch was set to record by one minute. Hourly PA and SB were calculated using the minutes' data. For all subjects, absolute time in moderate-to-vigorous physical activity (MVPA), walking steps, kcals in energy expenditure and absolute time spent in SB were estimated using the device. Nonwear hour periods were defined as 60 consecutive minutes of zero activity intensity count at 1 METs and 0 kcals in one hour [27].

\subsubsection{Environmental measures}

AP data and other environmental measures were selected including-air quality index (AQI), $\mathrm{PM}_{2.5}$, and average daytime temperature $\left({ }^{\circ} \mathrm{C}\right)$. Hourly $\mathrm{AQI}$ and $\mathrm{PM}_{2.5}$ data were provided by the Beijing Municipal Ecological Environment Bureau. The data collection site is Wan Liu was the Hai Dian district site, which is approximately $5 \mathrm{~km}$ from Tsinghua University. The air quality measurement data we collected are concurrent with PA measurements. Daytime temperature was retrieved from the China Meteorological Administration.

The AQI is an index for reporting daily air quality [28]. It indicates you how clean or polluted the surrounding air is, and which associated health effects might be of concern for you. The AQI focuses on health effects that may be experienced within a few hours or days after breathing polluted air. The China Meteorological Administration calculates the AQI using the following five major air pollutants: ground-level ozone, particle pollution (also known as particulate matter), carbon monoxide, sulfur dioxide, and nitrogen dioxide.

There are six levels of warning (a scale from 1 [green warning] to 6 [maroon warning]) on AP's health impacts based on AQI categories recommended by the Ministry of Ecology and Environment of China. There are six thresholds for AP in China [29] including 1: $0-50 \mu \mathrm{g} / \mathrm{m}^{3}$ (good, green warning); $2: 51-100 \mu \mathrm{g} / \mathrm{m}^{3}$ (fair, yellow warning); 3: $101-150 \mu \mathrm{g} / \mathrm{m}^{3}$ (mild pollution, orange warning); 4 : $151-200 \mu \mathrm{g} / \mathrm{m}^{3}$ (moderate pollution, red warning); 5: 201-300 $\mu \mathrm{g} / \mathrm{m}^{3}$ (severe pollution, purple warning); and 6: $>300 \mu \mathrm{g} / \mathrm{m}^{3}$ (serious pollution, maroon warning).

\subsection{Statistical Methods}

Descriptive statistics were computed including means, SD, and percentages. All descriptive statistics were compared for the characteristics of the overall sample. Chi-square tests were conducted to compare categorical variables. ANOVA tests and t-test were 
used to compare for continuous variables. One-way repeated measures ANOVA tests were conducted to compare the differences in hourly time between 7 am and $11 \mathrm{pm}$. Adjusted linear individual fixed-effect regressions were performed to reveal the associations between the accelerometer data and AP data. The data of participant controlled for age, gender, BMl, self-rated physical health, selfrated mental health, smoking, drinking, temperature, and temporal order for participants.

The key independent variables were AQI and $\mathrm{PM}_{2.5}$ during this time. We performed six levels (1-6) in AQI and used $10 \mu \mathrm{g} / \mathrm{m}^{3}$ in $\mathrm{PM} 2.5$ in the model analysis. Individual-level time-variant covariates and environmental measures including average daytime temperature were controlled for the aforementioned. Each outcome variable was then analyzed using separate regression and were stratified by specific time (from 7 am to $11 \mathrm{pm}$ ).

Compared to the conventional pooled cross-sectional regression, individual fixed-effect regression was selected due to its used of within-individual variations in hourly PA and SB to identify the impacts of AP concentration, thus removing potential omitted variable bias due to differences in time-invariant individual characteristics such as habits, and personal preferences.

\section{Results}

\subsection{Descriptive Statistics}

Table 1 presents the characteristics of the participants. Among the 340 student participants, accounting for more than two-thirds (70.6\%) were male. Participants complied to wearing a wGT3X accelerometer for 42291 hours over 7 continuous days. The mean age of the participants was $18.4(S D=1.0)$. The mean participants' BMI was $21.6 \mathrm{~kg} / \mathrm{m}^{2}(\mathrm{SD}=3.1)$ where male BMI was significantly higher than females' $(p<0.001)$. Only $0.88 \%$ of participants were reported smokers and $2.9 \%$ was reported drinkers. The mean selfrated physiological health score was $5.5(S D=1.8)$ and the mean self-rated mental health score was $6.5(S D=1.9)$.

Table 1

The characteristics of subjects

\begin{tabular}{|lllll|}
\hline Characteristics & Male & Female & Total & p \\
\hline Gender, $\mathrm{n}(\%)$ & $240(70.6)$ & $100(29.4)$ & 340 & $<0.001$ \\
\hline Compliance, hour & $31331(74.1)$ & $10960(25.9)$ & 42291 & $<0.001$ \\
\hline Age (yr), mean (SD) & $18.3(1.0)$ & $18.6(0.8)$ & $18.4(1.0)$ & 0.008 \\
\hline Height (cm), mean (SD) & $175.6(6.0)$ & $164.0(5.5)$ & $172.1(7.9)$ & $<0.001$ \\
\hline Weight (kg), mean (SD) & $68.2(11.5)$ & $55.4(6.7)$ & $64.3(11.9)$ & $<0.001$ \\
\hline Body mass index, mean (SD) & & & & \\
\hline BMI (kg/m $\left.{ }^{2}\right)$ & $22.1(3.2)$ & $20.6(2.4)$ & $21.6(3.1)$ & $<0.001$ \\
\hline Smoking, $\mathrm{n}$ (\%) & $3(1.3)$ & $0(0)$ & $3(0.9)$ & 0.001 \\
\hline Drinking $\mathrm{n}$ (\%) & $2(0.8)$ & $5(5.0)$ & $7(2.9)$ & 0.001 \\
\hline Self-rated physical health, mean (SD) & & & & \\
\hline Physical health score (1-10) & $5.5(1.8)$ & $5.6(1.8)$ & $5.5(1.8)$ & 0.928 \\
\hline Self-rated mental health, mean (SD) & & & & \\
\hline Mental health score (1-10) & $6.7(1.9)$ & $6.1(1.8)$ & $6.5(1.9)$ & 0.01 \\
\hline
\end{tabular}

\section{The AP Variations.}

Table 2 shows the variations of AP measures during the study period. The mean AQI value at one-hour was 106.1 (SD = 88.7). There were 10,977 (26.6\%), 14,980 (36.4\%), 6,476 (15.7\%), 2,329 (5.7\%), 4,868 (11.8\%) and 1,583 (3.8\%) one hour AQI at "good" AQI (0-50), "moderate" AQI (51-100), "Unhealthy for sensitive groups" AQI (101-150), "unhealth" AQI (151-200), "very unhealthy" AQI (201300 ) and "harzardous" AQI (>300), respectively. The mean concentration of $\mathrm{PM}_{2.5}$ values at one-hour was $68.0 \mu \mathrm{g} / \mathrm{m}^{3}(\mathrm{SD}=66.8)$. A 
majority proportion (59.1\%) of the average $\mathrm{PM}_{2.5}$ values were in concentrations that exceeded the U.S. Environmental Protection Agency (EPA) $\mathrm{PM}_{2.5}$ standard of $35 \mu \mathrm{g} / \mathrm{m}^{3}$. The mean temperature during the study was $5.5^{\circ} \mathrm{C}(\mathrm{SD}=5.6)$.

Table 2

Air quality index and PM2.5 categories during the study period by hours.

\begin{tabular}{|c|c|c|c|c|c|c|}
\hline & Level & Alarm & Levels of health concern & Value range & $\mathbf{N}$ & $\%$ \\
\hline & & & & & 41213 & 100 \\
\hline \multirow[t]{7}{*}{ AQI } & 1 & Green & Good & $0-50$ & 10,977 & 26.6 \\
\hline & 2 & Yellow & Moderate & $51-100$ & 14,980 & 36.4 \\
\hline & 3 & Orange & Unhealthy for sensitive groups & $101-150$ & 6,476 & 15.7 \\
\hline & 4 & Red & Unhealth & $151-200$ & 2,329 & 5.7 \\
\hline & 5 & Purple & Very unhealthy & $201-300$ & 4,868 & 11.8 \\
\hline & 6 & Maroon & Harzardous & $>300$ & 1,583 & 3.8 \\
\hline & & & & & 40947 & 100 \\
\hline PM2.5 & 1 & Green & Good & $0-12$ & 9,954 & 23.8 \\
\hline \multirow[t]{5}{*}{$\left(\mu \mathrm{g} / \mathrm{m}^{3}\right)$} & 2 & Yellow & Moderate & $12.1-35.4$ & 7,121 & 17.1 \\
\hline & 3 & Orange & Unhealthy for sensitive groups & $35.5-55.4$ & 6,700 & 16.1 \\
\hline & 4 & Red & Unhealth & $55.5-150.4$ & 11,816 & 28.3 \\
\hline & 5 & Purple & Very unhealthy & $150.5-250.4$ & 5,205 & 12.5 \\
\hline & 6 & Maroon & Harzardous & $>250.5$ & 958 & 2.3 \\
\hline \multirow[t]{6}{*}{ Temperature $\left({ }^{\circ} \mathrm{C}\right)$} & & & & & 41144 & 100 \\
\hline & & & & $-5-0$ & 5,397 & 13.1 \\
\hline & & & & $0.5-5$ & 20,019 & 48.7 \\
\hline & & & & $5.5-10$ & 7,332 & 17.8 \\
\hline & & & & $10.5-15$ & 4,182 & 10.2 \\
\hline & & & & $15.5-19$ & 4,214 & 10.2 \\
\hline
\end{tabular}

\section{The PA and SB Variations.}

Table 3 presents the mean variations of participants' PA and SB in the study. As illustrated, there are large variations in PA and SB. For example, the mean minutes of the participant's one-hour MVPA was $3.5(S D=4.5)$. One-hour mean minutes of MVPA ranged largely from $1.6(S D=2.7)$ at $11 \mathrm{pm}$ to $6.1(\mathrm{SD}=8.3)$ at $5 \mathrm{pm}$ in the participants $(\mathrm{p}<0.001)$. The mean steps of the participant's one-hour walking was $394(S D=627)$. Table 1 shows that one-hour mean steps of walking ranged largely from $147(S D=303)$ at $11 \mathrm{pm}$ to 710 $(S D=947)$ at $5 \mathrm{pm}$ in the participants $(p<0.001)$. Similarly, the mean participant's energy expenditure in one-hour was $19.8(S D=$ 39.1). One-hour mean energy expenditure in kcal ranged largely from $9.2(S D=19.5)$ at $11 \mathrm{pm}$ to $38.0(S D=66.2)$ at $5 \mathrm{pm}$ among the participants $(p<0.001)$. Whereas the mean minutes of participant's one-hour SB was $31.5(S D=19.7)$. One-hour mean minutes of SB ranged largely from $38.6(S D=18.2)$ at $11 \mathrm{pm}$ to $25.4(S D=17.1)$ at $12 \mathrm{pm}$ among the participants $(\mathrm{p}<0.001)$. 
Table 3

Average total and specific time of physical activity, sedentary behavior

\begin{tabular}{|c|c|c|c|c|c|c|c|c|}
\hline & $\begin{array}{l}M^{M V P A} A^{a} \text { mean } \\
\text { (SD) }\end{array}$ & $p$ & $\begin{array}{l}\text { Steps }{ }^{b} \text { mean } \\
\text { (SD) }\end{array}$ & $p$ & $\begin{array}{l}\mathrm{Kcals}^{\mathrm{C}} \text { mean } \\
(\mathrm{SD})\end{array}$ & $p$ & $\begin{array}{l}\mathrm{SB}^{\mathrm{d}} \text { mean } \\
\text { (SD) }\end{array}$ & $p$ \\
\hline \multicolumn{9}{|c|}{ Total average $P A^{e}$ and $S B$} \\
\hline & $3.5(4.5)$ & & $394(627)$ & & $19.8(39.1)$ & & $31.5(19.7)$ & \\
\hline $\begin{array}{l}\text { Specific Ho } \\
\text { SB }\end{array}$ & urly average $P A$ and & $<.001$ & & $<.001$ & & $\begin{array}{l}< \\
0.001\end{array}$ & & $\begin{array}{l}<.001 \\
0.00\end{array}$ \\
\hline 7:00 am & $4.6(3.8)$ & & $505(434)$ & & $24.4(23.5)$ & & $34.2(16.3)$ & \\
\hline $8: 00 \mathrm{am}$ & $2.3(4.1)$ & & $248(421)$ & & $12.2(26.2)$ & & $36.9(19.2)$ & \\
\hline 9:00 am & $4.4(3.9)$ & & $483(444)$ & & $22.8(23.4)$ & & $28.9(17.6)$ & \\
\hline 10:00 am & $2.0(4.6)$ & & $213(508)$ & & $11.2(30.6)$ & & $34.5(20.4)$ & \\
\hline 11:00 am & $3.7(4.8)$ & & $433(555)$ & & $19.2(29.3)$ & & $29.5(19.6)$ & \\
\hline $12: 00 \mathrm{pm}$ & $5.4(5.3)$ & & $630(589)$ & & $27.9(34.0)$ & & $25.4(17.1)$ & \\
\hline $1: 00 \mathrm{pm}$ & $4.1(5.2)$ & & $447(575)$ & & $22.2(35.5)$ & & $29.1(18.2)$ & \\
\hline $2: 00 \mathrm{pm}$ & $2.6(5.5)$ & & $270(558)$ & & $15.2(39.0)$ & & $32.6(20.8)$ & \\
\hline $3: 00 \mathrm{pm}$ & $4.1(6.4)$ & & $482(767)$ & & $24.7(49.7)$ & & $29.9(20.5)$ & \\
\hline $4: 00 \mathrm{pm}$ & $4.1(7.4)$ & & $460(823)$ & & $25.9(58.5)$ & & $29.1(20.5)$ & \\
\hline $5: 00 \mathrm{pm}$ & $6.1(8.3)$ & & $710(974)$ & & $38.0(66.2)$ & & $25.7(19.9)$ & \\
\hline $6: 00 \mathrm{pm}$ & $4.9(5.7)$ & & $572(645)$ & & $26.0(38.3)$ & & $25.9(17.9)$ & \\
\hline 7:00 pm & $3.2(5.0)$ & & 355 (569) & & $17.1(33.6)$ & & $30.4(19.2)$ & \\
\hline $8: 00 \mathrm{pm}$ & $2.3(5.1)$ & & $258(575)$ & & $13.2(35.0)$ & & $31.4(19.8)$ & \\
\hline 9:00 pm & $3.8(5.8)$ & & $416(664)$ & & $21.6(41.9)$ & & $28.6(19.2)$ & \\
\hline $10: 00 \mathrm{pm}$ & $3.1(4.7)$ & & 321 (589) & & $17.8(35.3)$ & & $31.3(19.0)$ & \\
\hline 11:00 pm & $1.6(2.7)$ & & 147 (303) & & 9.2 (19.5) & & $38.6(18.2)$ & \\
\hline
\end{tabular}

${ }^{a}$ Moderate-to-vigorous physical activity; ${ }^{b}$ Walking steps in one hour; ${ }^{C}$ Engery expenditure in kcals in one hours; ${ }^{\text {s Sedentary }}$ behavior; ePhysical activity.

\subsection{The Relationship between AP and PA, SB.}

Impact of AQI on PA and SB.

Table 4 shows the estimated effects of air quality index (AQI) on individual-level outcomes of hourly PA and SB using linear individual fixed-effect regressions. AQI was found to be significant and negatively associated with one-hour PA among participants. A one level increase in AQI was linked to a significant reduction in minutes of one-hour MVPA, in steps of one-hour walking, and in kcals of onehour energy expenditure by $0.083(95 \%$ confidence interval $[\mathrm{Cl}]=[-0.137,-0.029]), 8.8(95 \% \mathrm{Cl}=[-15.0,-2.6]), 0.653(95 \% \mathrm{Cl}=[-1.0$, $-0.3])$, respectively $(p<0.001)$. 
Table 4. Estimated effects of hourly AQI on individual-level one-hour PA and SB outcomes.

\begin{tabular}{|c|c|c|c|c|c|c|c|c|}
\hline \multirow[t]{2}{*}{ Effects } & \multicolumn{2}{|l|}{ MVPA } & \multicolumn{2}{|l|}{ Steps } & \multicolumn{2}{|l|}{ Kcals } & \multicolumn{2}{|l|}{ SB } \\
\hline & $\begin{array}{l}\text { Coefficient } \\
(95 \% \mathrm{Cl})\end{array}$ & $\begin{array}{l}\# \\
\text { Observations } \\
\text { (\# } \\
\text { participants) }\end{array}$ & $\begin{array}{l}\text { Coefficient } \\
(95 \% \mathrm{Cl})\end{array}$ & $\begin{array}{l}\text { \# } \\
\text { Observations } \\
\text { (\# } \\
\text { participants) }\end{array}$ & $\begin{array}{l}\text { Coefficient } \\
(95 \% \mathrm{Cl})\end{array}$ & $\begin{array}{l}\text { \# } \\
\text { Observations } \\
\text { (\# } \\
\text { participants) }\end{array}$ & $\begin{array}{l}\text { Coefficient } \\
(95 \% \mathrm{Cl})\end{array}$ & $\begin{array}{l}\# \\
\text { Observations } \\
\text { (\# } \\
\text { participants) }\end{array}$ \\
\hline \multicolumn{9}{|c|}{ Total effects } \\
\hline & $-0.083^{* *}$ & $40,275(340)$ & $-8.8^{* *}$ & $40,275(340)$ & $-0.65^{* *}$ & $40,275(340)$ & -0.085 & $40,275(340)$ \\
\hline & $\begin{array}{l}(-0.137 \\
-0.029)\end{array}$ & & $\begin{array}{l}(-15.0 \\
-2.6)\end{array}$ & & $\begin{array}{l}(-1.03 \\
-0.27)\end{array}$ & & $\begin{array}{l}(-0.271 \\
0.101)\end{array}$ & \\
\hline \multicolumn{9}{|c|}{$\begin{array}{l}\text { Specific times } \\
\text { effects }\end{array}$} \\
\hline \multirow{2}{*}{$\begin{array}{l}7: 00 \\
a m\end{array}$} & 0.063 & $1,362(316)$ & 6.3 & $1,362(316)$ & -0.18 & $1,362(316)$ & -0.170 & $1,362(316)$ \\
\hline & $\begin{array}{l}(-0.138 \\
0.264)\end{array}$ & & $\begin{array}{l}(-16.8 \\
29.5)\end{array}$ & & $\begin{array}{l}(-0.96 \\
1.32)\end{array}$ & & $\begin{array}{l}(-1.107 \\
0.767)\end{array}$ & \\
\hline \multirow{2}{*}{$\begin{array}{l}8: 00 \\
a m\end{array}$} & $-0.397^{\star \star}$ & 1,750 (331) & $-35.3^{\star \star}$ & $1,750(331)$ & $-2.37^{\star *}$ & $1,750(331)$ & $1.021^{*}$ & $1,750(331)$ \\
\hline & $\begin{array}{l}(-0.635 \\
-0.159)\end{array}$ & & $\begin{array}{l}(-59.5 \\
-11.2)\end{array}$ & & $(-3.9,0.83)$ & & $\begin{array}{l}(0.013 \\
2.028)\end{array}$ & \\
\hline \multirow{2}{*}{$\begin{array}{l}\text { 9:00 } \\
a m\end{array}$} & 0.045 & 2,165 (339) & -1.2 & 2,165 (339) & 0.93 & 2,165 (339) & $1.134^{* *}$ & 2,165 (339) \\
\hline & $\begin{array}{l}(-0.146 \\
0.236)\end{array}$ & & $\begin{array}{l}(-22.8 \\
20.4)\end{array}$ & & $\begin{array}{l}(-0.16 \\
2.01)\end{array}$ & & $\begin{array}{l}(0.308 \\
1.960)\end{array}$ & \\
\hline \multirow{2}{*}{$\begin{array}{l}10: 00 \\
a m\end{array}$} & 0.209 & $2,116(338)$ & 18.9 & $2,116(338)$ & $1.85^{\star}$ & 2,116 (338) & -0.188 & 2,116 (338) \\
\hline & $\begin{array}{l}(-0.035 \\
0.453)\end{array}$ & & $(-7.9,45.7)$ & & $\begin{array}{l}(0.24 \\
3.47)\end{array}$ & & $\begin{array}{l}(-1.190 \\
0.814)\end{array}$ & \\
\hline \multirow{2}{*}{$\begin{array}{l}\text { 11:00 } \\
a m\end{array}$} & -0.128 & $2,266(339)$ & -13.4 & $2,266(339)$ & -0.60 & 2,266 (339) & $1.480^{\star *}$ & 2,266 (339) \\
\hline & $\begin{array}{l}(-0.372 \\
0.116)\end{array}$ & & $\begin{array}{l}(-41.2 \\
14.5)\end{array}$ & & $\begin{array}{l}(-2.06 \\
0.86)\end{array}$ & & $\begin{array}{l}(0.557 \\
2.403)\end{array}$ & \\
\hline \multirow{2}{*}{$\begin{array}{l}\text { 12:00 } \\
\mathrm{pm}\end{array}$} & 0.072 & 2,399 (339) & 6.6 & 2,399 (339) & 0.27 & 2,399 (339) & 0.162 & 2,399 (339) \\
\hline & $\begin{array}{l}(-0.176 \\
0.319)\end{array}$ & & $\begin{array}{l}(-20.8 \\
34.0)\end{array}$ & & $\begin{array}{l}(-1.26 \\
1.80)\end{array}$ & & $\begin{array}{l}(-0.615 \\
0.938)\end{array}$ & \\
\hline \multirow{2}{*}{$\begin{array}{l}\text { 1:00 } \\
\mathrm{pm}\end{array}$} & -0.107 & $2,400(340)$ & 0.4 & $2,400(340)$ & -0.50 & $2,400(340)$ & 0.620 & $2,400(340)$ \\
\hline & $\begin{array}{l}(-0.337 \\
0.123)\end{array}$ & & $\begin{array}{l}(-24.7 \\
25.6)\end{array}$ & & $\begin{array}{l}(-2.03 \\
1.02)\end{array}$ & & $\begin{array}{l}(-0.159 \\
1.400)\end{array}$ & \\
\hline \multirow{2}{*}{$\begin{array}{l}2: 00 \\
p m\end{array}$} & 0.126 & 2,297 (339) & 19.2 & 2,297 (339) & 0.83 & 2,297 (339) & 0.150 & 2,297 (339) \\
\hline & $\begin{array}{l}(-0.107 \\
0.358)\end{array}$ & & $(-5.0,43.3)$ & & $\begin{array}{l}(-0.78 \\
2.43)\end{array}$ & & $\begin{array}{l}(-0.725 \\
1.025)\end{array}$ & \\
\hline
\end{tabular}




\begin{tabular}{|c|c|c|c|c|c|c|c|c|}
\hline 3:00 pm & $\begin{array}{l}0.258 * \\
(0.025 \\
0.491)\end{array}$ & $\begin{array}{l}2,538 \\
(340)\end{array}$ & $\begin{array}{l}28.6^{\star} \\
(0.8,56.4)\end{array}$ & $\begin{array}{l}2,538 \\
(340)\end{array}$ & $\begin{array}{l}0.81 \\
(0.96,2.58)\end{array}$ & $\begin{array}{l}2,538 \\
(340)\end{array}$ & $\begin{array}{l}-2.510 * \star \star \\
(-3.215 \\
-1.806)\end{array}$ & $\begin{array}{l}2,538 \\
(340)\end{array}$ \\
\hline $4: 00 \mathrm{pm}$ & 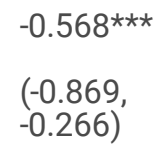 & $\begin{array}{l}2,455 \\
(340)\end{array}$ & $\begin{array}{l}-74.7^{\star \star \star} \\
(-108.3,-41.2)\end{array}$ & $\begin{array}{l}2,455 \\
(340)\end{array}$ & $\begin{array}{l}-4.79 \star * \star \\
(-7.13 \\
-2.44)\end{array}$ & $\begin{array}{l}2,455 \\
(340)\end{array}$ & $\begin{array}{l}-0.040 \\
(-0.840 \\
0.760)\end{array}$ & $\begin{array}{l}2,455 \\
(340)\end{array}$ \\
\hline $5: 00 \mathrm{pm}$ & $\begin{array}{l}-0.330 * \\
(-0.637 \\
-0.023)\end{array}$ & $\begin{array}{l}2,502 \\
(340)\end{array}$ & $\begin{array}{l}-35.7 \\
(-71.7,0.3)\end{array}$ & $\begin{array}{l}2,502 \\
(340)\end{array}$ & $\begin{array}{l}-2.53^{\star} \\
(-4.91 \\
-0.15)\end{array}$ & $\begin{array}{l}2,502 \\
(340)\end{array}$ & $\begin{array}{l}0.370 \\
(-0.362, \\
1.102)\end{array}$ & $\begin{array}{l}2,502 \\
(340)\end{array}$ \\
\hline $6: 00 \mathrm{pm}$ & $\begin{array}{l}-0.168 \\
(-0.374 \\
0.038)\end{array}$ & $\begin{array}{l}2,465 \\
(339)\end{array}$ & $\begin{array}{l}-18.6 \\
(-42.1,4.8)\end{array}$ & $\begin{array}{l}2,465 \\
(339)\end{array}$ & $\begin{array}{l}-1.09 \\
(-2.46 \\
0.28)\end{array}$ & $\begin{array}{l}2,465 \\
(339)\end{array}$ & $\begin{array}{l}0.293 \\
(-0.329 \\
0.915)\end{array}$ & $\begin{array}{l}2,465 \\
(339)\end{array}$ \\
\hline 7:00 pm & $\begin{array}{l}-0.234^{*} \\
(-0.430 \\
-0.039)\end{array}$ & $\begin{array}{l}2,389 \\
(339)\end{array}$ & $\begin{array}{l}-23.5^{\star} \\
(-45.7,-1.4)\end{array}$ & $\begin{array}{l}2,389 \\
(339)\end{array}$ & $\begin{array}{l}-1.41 * \\
(-2.72 \\
-0.10)\end{array}$ & $\begin{array}{l}2,389 \\
(339)\end{array}$ & $\begin{array}{l}0.884^{\star} \\
(0.177 \\
1.590)\end{array}$ & $\begin{array}{l}2,389 \\
(339)\end{array}$ \\
\hline $8: 00 p m$ & $\begin{array}{l}-0.078 \\
(-0.312 \\
0.156)\end{array}$ & $\begin{array}{l}2,262 \\
(338)\end{array}$ & $\begin{array}{l}-14.5 \\
(-41.3,12.4)\end{array}$ & $\begin{array}{l}2,262 \\
(338)\end{array}$ & $\begin{array}{l}-1.33 \\
(-2.97 \\
0.30)\end{array}$ & $\begin{array}{l}2,262 \\
(338)\end{array}$ & $\begin{array}{l}0.008 \\
(-0.817 \\
0.833)\end{array}$ & $\begin{array}{l}2,262 \\
(338)\end{array}$ \\
\hline 9:00 pm & $\begin{array}{l}-0.197 \\
(-0.430 \\
0.036)\end{array}$ & $\begin{array}{l}2,324 \\
(337)\end{array}$ & $\begin{array}{l}-20.2 \\
(-47.2,6.8)\end{array}$ & $\begin{array}{l}2,324 \\
(337)\end{array}$ & $\begin{array}{l}-1.65 \\
(-3.34 \\
0.04)\end{array}$ & $\begin{array}{l}2,324 \\
(337)\end{array}$ & $\begin{array}{l}0.148 \\
(-0.575 \\
0.872)\end{array}$ & $\begin{array}{l}2,324 \\
(337)\end{array}$ \\
\hline $\begin{array}{l}10: 00 \\
p m\end{array}$ & $\begin{array}{l}-0.067 \\
(-0.264 \\
0.131)\end{array}$ & $\begin{array}{l}2,163 \\
(337)\end{array}$ & $\begin{array}{l}-18.3 \\
(-43.1,6.5)\end{array}$ & $\begin{array}{l}2,163 \\
(337)\end{array}$ & $\begin{array}{l}-0.53 \\
(-1.99 \\
0.92)\end{array}$ & $\begin{array}{l}2,163 \\
(337)\end{array}$ & $\begin{array}{l}-0.068 \\
(-0.845 \\
0.709)\end{array}$ & $\begin{array}{l}2,163 \\
(337)\end{array}$ \\
\hline $\begin{array}{l}\text { 11:00 } \\
\text { pm }\end{array}$ & $\begin{array}{l}0.045 \\
(-0.085 \\
0.175)\end{array}$ & $\begin{array}{l}1,768 \\
(331)\end{array}$ & $\begin{array}{l}5.9 \\
(-9.0,20.7)\end{array}$ & $\begin{array}{l}1,768 \\
(331)\end{array}$ & $\begin{array}{l}0.67 \\
(-0.28 \\
1.63)\end{array}$ & $\begin{array}{l}1,768 \\
(331)\end{array}$ & $\begin{array}{l}-0.059 \\
(-0.889 \\
0.770)\end{array}$ & $\begin{array}{l}1,768 \\
(331)\end{array}$ \\
\hline
\end{tabular}

Notes: Separate individual fixed-effect regressions were performed to estimate the effects of hourly AP concentrations on samples stratified by specific time. Models adjust for all time-variant individual characteristics listed in Table 1 (i.e., age, gender, BMI, drinking status, drinking status, self-rated physical health, self-rated mental health, temporal order for participants) and environmental variables (average temperature). ${ }^{*} P<0.05 ;{ }^{* *} P<0.01 ;{ }^{* *} P<0.001$

The impact of AQI on individual-level one-hour PA at a specific time was different. AQI was found to be more negatively associated with participants' one-hour PA at 8 am, 4 pm, 5 pm and $7 \mathrm{pm}$. Specifically, a one level increase in AQI was linked with a significantly reduction in steps of one-hour walking at $8 \mathrm{am}, 4 \mathrm{pm}$ and $7 \mathrm{pm}$ by $35.3(95 \% \mathrm{Cl}=[-59.5,-11.2]), 74.7(95 \% \mathrm{Cl}=[-108.3,-41.2])$, and $23.5(95 \% \mathrm{Cl}=[-45.7,-1.4])$, respectively. However, AQI was found to be positively associated with participants' one-hour PA at 10 am and $3 \mathrm{pm}$. There was no significant relationship between AQI and one-hour SB among participants.

\section{Impact of $\mathrm{PM}_{2.5}$ on PA and SB.}

Table 5 indicates the estimated effects of AP concentration in $\mathrm{PM}_{2.5}$ on individual-level outcomes of hourly PA and SB using linear individual fixed-effect regressions. $\mathrm{PM}_{2.5}$ was found to be significantly negative associated with one-hour PA among participants. A $10 \mu \mathrm{g} / \mathrm{m}^{3}$ increase in $\mathrm{PM}_{2.5}$ was associated with a significant reduction in minutes of one-hour MVPA, in steps of one-hour walking, and in kcals of one-hour energy expenditure by $0.021(95 \% \mathrm{Cl}=-0.033,-0.010), 2.2(95 \% \mathrm{Cl}=[-3.5,-0.9])$ and $0.170(95 \% \mathrm{Cl}=[-0.250$, $-0.089])$, respectively $(p<0.001)$. 
Table 5. Estimated effects of hourly $\mathrm{PM}_{2.5}$ on individual-level one-hour PA and SB outcomes.

\begin{tabular}{|c|c|c|c|c|c|c|c|c|}
\hline \multirow[t]{2}{*}{ Effects } & \multicolumn{2}{|l|}{ MVPA } & \multicolumn{2}{|l|}{ Steps } & \multicolumn{2}{|l|}{ Kcals } & \multicolumn{2}{|l|}{ SB } \\
\hline & $\begin{array}{l}\text { Coefficient } \\
(95 \% \mathrm{Cl})\end{array}$ & $\begin{array}{l}\# \\
\text { Observations } \\
\text { (\# } \\
\text { participants) }\end{array}$ & $\begin{array}{l}\text { Coefficient } \\
(95 \% \mathrm{Cl})\end{array}$ & $\begin{array}{l}\text { \# } \\
\text { Observations } \\
\text { (\# } \\
\text { participants) }\end{array}$ & $\begin{array}{l}\text { Coefficient } \\
(95 \% \mathrm{Cl})\end{array}$ & $\begin{array}{l}\text { \# } \\
\text { Observations } \\
\text { (\# } \\
\text { participants) }\end{array}$ & $\begin{array}{l}\text { Coefficient } \\
(95 \% \mathrm{Cl})\end{array}$ & $\begin{array}{l}\text { \# } \\
\text { Observations } \\
\text { (\# } \\
\text { participants) }\end{array}$ \\
\hline \multicolumn{9}{|c|}{ Total effects } \\
\hline & $-0.021^{\star \star \star}$ & $40,009(340)$ & $-2.2^{* \star}$ & $40,009(340)$ & $-0.170^{\star \star \star}$ & $40,009(340)$ & $0.045^{*}$ & $40,009(340)$ \\
\hline & $\begin{array}{l}(-0.033 \\
-0.010)\end{array}$ & & $(-3.5,-0.9)$ & & $\begin{array}{l}(-0.250 \\
-0.089)\end{array}$ & & $\begin{array}{l}(0.005 \\
0.0845)\end{array}$ & \\
\hline \multicolumn{9}{|c|}{ Times effects } \\
\hline \multirow{2}{*}{$\begin{array}{l}7: 00 \\
a m\end{array}$} & 0.037 & $1,362(316)$ & 4.9 & $1,362(316)$ & 0.168 & $1,362(316)$ & -0.073 & $1,362(316)$ \\
\hline & $\begin{array}{l}(-0.010 \\
0.084)\end{array}$ & & $(-0.5,10.3)$ & & $\begin{array}{l}(-0.097 \\
0.434)\end{array}$ & & $\begin{array}{l}(-0.292 \\
0.146)\end{array}$ & \\
\hline \multirow[t]{2}{*}{$\begin{array}{l}8: 00 \\
a m\end{array}$} & $-0.069^{\star \star}$ & $1,750(331)$ & $-6.1^{*}$ & $1,750(331)$ & $-0.397^{\star}$ & $1,750(331)$ & 0.154 & $1,750(331)$ \\
\hline & $\begin{array}{l}(-0.120 \\
-0.019)\end{array}$ & & $\begin{array}{l}(-11.3 \\
-1.0)\end{array}$ & & $\begin{array}{l}(-0.723, \\
-0.070)\end{array}$ & & $\begin{array}{l}(-0.061, \\
0.369)\end{array}$ & \\
\hline \multirow{2}{*}{$\begin{array}{l}9: 00 \\
a m\end{array}$} & -0.005 & 2,165 (339) & -1.8 & 2,165 (339) & 0.079 & 2,165 (339) & $0.247^{\star \star}$ & 2,165 (339) \\
\hline & $\begin{array}{l}(-0.047 \\
0.036)\end{array}$ & & $(-6.5,2.9)$ & & $\begin{array}{l}(-0.158, \\
0.316)\end{array}$ & & $\begin{array}{l}(0.067 \\
0.427)^{\prime}\end{array}$ & \\
\hline \multirow{2}{*}{$\begin{array}{l}10: 00 \\
a m\end{array}$} & $0.101^{\star \star *}$ & 2,116 (338) & $9.5^{\star \star}$ & 2,116 (338) & $0.786^{\star \star \star}$ & $2,116(338)$ & -0.133 & 2,116 (338) \\
\hline & $\begin{array}{l}(0.047 \\
0.155)\end{array}$ & & $(3.6,15.5)$ & & $\begin{array}{l}(0.428, \\
1.144)\end{array}$ & & $\begin{array}{l}(-0.356 \\
0.090)\end{array}$ & \\
\hline \multirow{2}{*}{$\begin{array}{l}11: 00 \\
a m\end{array}$} & -0.029 & 2,216 (339) & -4.9 & 2,216 (339) & -0.137 & 2,216 (339) & $0.342^{\star *}$ & 2,216 (339) \\
\hline & $\begin{array}{l}(-0.083 \\
0.024)\end{array}$ & & $(-11 ., 1.2)$ & & $\begin{array}{l}(-0.458, \\
0.185)\end{array}$ & & $\begin{array}{l}(0.139 \\
0.544)\end{array}$ & \\
\hline \multirow{2}{*}{$\begin{array}{l}\text { 12:00 } \\
p m\end{array}$} & -0.004 & 2,390 (339) & 0.0 & 2,390 (339) & -0.025 & 2,390 (339) & 0.072 & 2,390 (339) \\
\hline & $\begin{array}{l}(-0.056 \\
0.047)\end{array}$ & & $(-5.7,5.8)$ & & $\begin{array}{l}(-0.345 \\
0.295)\end{array}$ & & $\begin{array}{l}(-0.091, \\
0.234)\end{array}$ & \\
\hline \multirow{2}{*}{$\begin{array}{l}1: 00 \\
p m\end{array}$} & -0.019 & 2,400 (339) & 0.6 & 2,400 (339) & -0.079 & 2,400 (339) & 0.131 & 2,400 (339) \\
\hline & $\begin{array}{l}(-0.068 \\
0.030)\end{array}$ & & $(-4.9,5.8)$ & & $\begin{array}{l}(-0.403, \\
0.246)\end{array}$ & & $\begin{array}{l}(-0.035 \\
0.297)\end{array}$ & \\
\hline \multirow{2}{*}{$\begin{array}{l}2: 00 \\
p m\end{array}$} & 0.039 & 2,297 (339) & 5.3 & 2,297 (339) & 0.286 & 2,297 (339) & 0.116 & 2,297 (339) \\
\hline & $\begin{array}{l}(-0.013 \\
0.091)\end{array}$ & & $(-0.0,10.7)$ & & $\begin{array}{l}(-0.071 \\
0.643)\end{array}$ & & $\begin{array}{l}(-0.078, \\
0.311)\end{array}$ & \\
\hline
\end{tabular}




\begin{tabular}{|c|c|c|c|c|c|c|c|c|}
\hline $3: 00 \mathrm{pm}$ & $\begin{array}{l}0.052^{\star} \\
(0.002 \\
0.102)\end{array}$ & $\begin{array}{l}2,538 \\
(340)\end{array}$ & $\begin{array}{l}5.6 \\
(-0.4 \\
11.6)\end{array}$ & $\begin{array}{l}2,538 \\
(340)\end{array}$ & $\begin{array}{l}0.165 \\
(-0.216 \\
0.547)\end{array}$ & $\begin{array}{l}2,538 \\
(340)\end{array}$ & $\begin{array}{l}-0.455^{\star \star \star} \\
(-0.608 \\
-0.303)\end{array}$ & $\begin{array}{l}2,538 \\
(340)\end{array}$ \\
\hline 4:00 pm & $\begin{array}{l}-0.094 \text { ** } \\
(-0.153 \\
-0.034)\end{array}$ & $\begin{array}{l}2,400 \\
(340)\end{array}$ & $\begin{array}{l}-10.9 * \star \\
(-17.4 \\
-4.3)\end{array}$ & $\begin{array}{l}2,400 \\
(340)\end{array}$ & $\begin{array}{l}-0.781 \text { ** } \\
(-1.242 \\
-0.320)\end{array}$ & $\begin{array}{l}2,400 \\
(340)\end{array}$ & $\begin{array}{l}0.037 \\
(-0.129 \\
0.203)\end{array}$ & $\begin{array}{l}2,400 \\
(340)\end{array}$ \\
\hline $5: 00 \mathrm{pm}$ & $\begin{array}{l}-0.082^{\star} \\
(-0.151 \\
-0.014)\end{array}$ & $\begin{array}{l}2,502 \\
(340)\end{array}$ & $\begin{array}{l}-9.7{ }^{\star} \\
(-17.6 \\
-1.7)\end{array}$ & $\begin{array}{l}2,502 \\
(340)\end{array}$ & $\begin{array}{l}-0.690 * \\
(-1.218 \\
-0.162)\end{array}$ & $\begin{array}{l}2,502 \\
(340)\end{array}$ & $\begin{array}{l}0.192^{\star} \\
(0.030,0.355)\end{array}$ & $\begin{array}{l}2,502 \\
(340)\end{array}$ \\
\hline $6: 00 \mathrm{pm}$ & $\begin{array}{l}-0.033 \\
(-0.079 \\
0.013)\end{array}$ & $\begin{array}{l}2,465 \\
(339)\end{array}$ & $\begin{array}{l}-3.8 \\
(-9.1,1.5)\end{array}$ & $\begin{array}{l}2,465 \\
(339)\end{array}$ & $\begin{array}{l}-0.263 \\
(-0.572 \\
0.046)\end{array}$ & $\begin{array}{l}2,465 \\
(339)\end{array}$ & $\begin{array}{l}0.090 \\
(-0.050 \\
0.230)\end{array}$ & $\begin{array}{l}2,465 \\
(339)\end{array}$ \\
\hline 7:00 pm & $\begin{array}{l}-0.049 * \\
(-0.092 \\
-0.007)\end{array}$ & $\begin{array}{l}2,340 \\
(339)\end{array}$ & $\begin{array}{l}-4.8^{\star} \\
(-9.6,0.0)\end{array}$ & $\begin{array}{l}2,340 \\
(339)\end{array}$ & $\begin{array}{l}-0.291^{\star} \\
(-0.574 \\
-0.008)\end{array}$ & $\begin{array}{l}2,340 \\
(339)\end{array}$ & $\begin{array}{l}0.201^{\star} \\
(0.048,0.355)\end{array}$ & $\begin{array}{l}2,340 \\
(339)\end{array}$ \\
\hline 8:00 pm & $\begin{array}{l}-0.027 \\
(-0.069 \\
0.015)\end{array}$ & $\begin{array}{l}2,212 \\
(338)\end{array}$ & $\begin{array}{l}-3.0 \\
(-7.9,1.9)\end{array}$ & $\begin{array}{l}2,212 \\
(338)\end{array}$ & $\begin{array}{l}-0.289 \\
(-0.579 \\
0.000)\end{array}$ & $\begin{array}{l}2,212 \\
(338)\end{array}$ & $\begin{array}{l}0.024 \\
(-0.130 \\
0.179)\end{array}$ & $\begin{array}{l}2,212 \\
(338)\end{array}$ \\
\hline 9:00 pm & $\begin{array}{l}-0.020 \\
(-0.065 \\
0.025)\end{array}$ & $\begin{array}{l}2,271 \\
(337)\end{array}$ & $\begin{array}{l}-0.4 \\
(-5.6,4.7)\end{array}$ & $\begin{array}{l}2,271 \\
(337)\end{array}$ & $\begin{array}{l}-0.222 \\
(-0.549 \\
-0.105)\end{array}$ & $\begin{array}{l}2,271 \\
(337)\end{array}$ & $\begin{array}{l}-0.003 \\
(-0.144 \\
0.139)\end{array}$ & $\begin{array}{l}2,271 \\
(337)\end{array}$ \\
\hline $\begin{array}{l}\text { 10:00 } \\
p m\end{array}$ & $\begin{array}{l}-0.007 \\
(-0.045 \\
0.030)\end{array}$ & $\begin{array}{l}2,163 \\
(337)\end{array}$ & $\begin{array}{l}-2.3 \\
(-7.0,2.5)\end{array}$ & $\begin{array}{l}2,163 \\
(337)\end{array}$ & $\begin{array}{l}-0.087 \\
(-0.363 \\
0.190)\end{array}$ & $\begin{array}{l}2,163 \\
(337)\end{array}$ & $\begin{array}{l}0.047 \\
(-0.101 \\
0.194)\end{array}$ & $\begin{array}{l}2,163 \\
(337)\end{array}$ \\
\hline $\begin{array}{l}\text { 11:00 } \\
\text { pm }\end{array}$ & $\begin{array}{l}0.016 \\
(-0.010 \\
0.042)\end{array}$ & $\begin{array}{l}1,768 \\
(331)\end{array}$ & $\begin{array}{l}1.9 \\
(-1.1,4.9)\end{array}$ & $\begin{array}{l}1,768 \\
(331)\end{array}$ & $\begin{array}{l}0.174 \\
(-0.017 \\
0.364)\end{array}$ & $\begin{array}{l}1,768 \\
(331)\end{array}$ & $\begin{array}{l}-0.045 \\
(-0.211 \\
0.121)\end{array}$ & $\begin{array}{l}1,768 \\
(331)\end{array}$ \\
\hline
\end{tabular}

The impact of $\mathrm{PM}_{2.5}$ on individual-level one-hour $\mathrm{PA}$ at specific time was also different. $\mathrm{PM}_{2.5}$ was found to be more negatively associated with participants' one-hour PA at 8 am, 4 pm, 5 pm and 7 pm. Specifically, a $10 \mu \mathrm{g} / \mathrm{m}^{3}$ increase in PM 2.5 was linked with a significantly reduction in steps of one-hour walking at $8 \mathrm{am}, 4 \mathrm{pm}, 5 \mathrm{pm}$ and $7 \mathrm{pm}$ by $6.1(95 \% \mathrm{Cl}=[-11.3,-1.0]), 10.9(95 \% \mathrm{Cl}=[-17.4$, $-4.3])$ and $9.7(95 \% \mathrm{Cl}=[-17.6,-1.7])$ and $5.0(95 \% \mathrm{Cl}=[-9.6,-0.0])$, respectively. However, $\mathrm{PM}_{2.5}$ was found to be positively associated with participants' one-hour PA at $10 \mathrm{am}, 2 \mathrm{pm}$ and $3 \mathrm{pm}$. AP concentration in $\mathrm{PM}_{2.5}$ was found to be significantly positive associated with SB among participants. A $10 \mu \mathrm{g} / \mathrm{m}^{3}$ increase in $\mathrm{PM}_{2.5}$ was associated with a significant increase in minutes of one-hour $\mathrm{SB}$ by $0.045(95 \% \mathrm{Cl}=[-0.005,-0.0845])$.

The impact of $\mathrm{PM}_{2.5}$ on individual-level one-hour $\mathrm{SB}$ at a specific time was also different. $\mathrm{PM}_{2.5}$ was found to be more positively associated with participants' one-hour SB at 9 am, 11 pm, $5 \mathrm{pm}$ and $7 \mathrm{pm}$. Specifically, a $10 \mu \mathrm{g} / \mathrm{m}^{3}$ increase in PM 2.5 was linked with a significantly increase in minutes of one-hour SB at $9 \mathrm{am}, 11 \mathrm{pm}, 5 \mathrm{pm}$ and $7 \mathrm{pm}$ by $0.247(95 \% \mathrm{Cl}=[0.067,0.427]), 0.342(95 \% \mathrm{Cl}=$ $[0.139,0.544]), 0.192(95 \% \mathrm{Cl}=[0.030,0.355])$ and $0.201(95 \% \mathrm{Cl}=[0.048,0.355])$, respectively. However, $\mathrm{PM}_{2.5}$ was found to be negatively associated with participants' one-hour PA at 10 am and $3 \mathrm{pm}$.

\section{Discussion}


The purpose of this study was to examine the impact of AP level on PA and SB among university freshman students in Beijing, China from November 2017 to April 2018 using objectively-measured PA and SB. Our study found a significantly negative relationship between AP and PA and a positive relationship between AP and SB. With a one level increase in AQI and a $10 \mu \mathrm{g} / \mathrm{m}^{3}$ increase in $\mathrm{PM} 2.5$, hourly total minutes of MVPA, walking steps and kcals of energy expenditure were significantly reduced. A $10 \mu \mathrm{g} / \mathrm{m}^{3}$ increase in $\mathrm{PM}_{2.5}$ was associated with a significantly increase in SB among participants. The impact of AP on individual-level one-hour PA and SB behavior at a specific time was different. To our best knowledge, this is the first study to use objective methods to determine the effect of hourly AP on PA and SB. In addition, this is the first study to estimate the impact of AP on PA and SB at a specific time.

Our findings on the negative relationship between AP and PA are consistent with existing literature [19, 30-33]. In our study, we found that a one-hour AQI increase one level was associated with a decrease by 9 walking steps in one hour. This study additionally found that a $10 \mu \mathrm{g} / \mathrm{m}^{3}$ increase in $\mathrm{PM}_{2.5}$ was linked with reduction by 2 walking steps in one hour. Two previous U.S. studies linked one unit $\left(<10 \mu \mathrm{g} / \mathrm{m}^{3}\right)$ monthly average $\mathrm{PM}_{2.5}$ increase of AP to be associated with decreasing $0.46 \%$ leisure time PA using a cross sectional study from the US Behavioral Risk Factor Surveillance System (BRFSS) survey [31, 34]. Evidence from our previous follow-up studies also found a one unit (44.72-56.6 $\left.\mathrm{\mu g} / \mathrm{m}^{3}\right)$ increase in $\mathrm{PM}_{2.5}$ to discourage outdoor PA 110.67 PASE scores among older adults in China [30] and to reduce 32.45 weekly MVPA among Chinese college students [19, 35]. However, these previous studies were limited by the potential social bias of self-report measures of PA and therefore were have not been able to examine the hourly effects of AP on objective PA. Only two studies with objectively measured data have reported that the association between AP and PA. Consistent with this study, a study of 153 middle-age adult users of an exercise app reported that AQI increase was associated with participates' reduction in outdoor PA, such as running, biking, and walking [33]. With this current analysis, we can more precisely use accelerometers to estimate the impact of $A Q I$ and $\mathrm{PM}_{2.5}$ on MVPA, energy expenditure, and steps rather than use an exercise app associated with PA. Inconsistent with this study, another study showed that $\mathrm{PM}_{2.5}$ increase had no impact on PA in Beijing among 40 Han Chinese participants in the mean age of 31 years using GT3X accelerometers [36]. A possible explanation for this difference could be that the study had a relatively small sample size and could not account for differences among participants. Based on 340 participants' WGT3X accelerometers data, we can more confidently suggest that an increase in AQI and PM 2.5 increase was associated with a reduction of PA in MVPA, energy expenditure, and walking steps.

This study confirmed findings from previous studies regarding the positive correlation between AP and SB [37-39]. This finding suggests that a one-hour $10 \mu \mathrm{g} / \mathrm{m}^{3} \mathrm{PM}_{2.5}$ increase was associated with an increase in SB by 0.045 minutes in one hour. Consistent with our previous research, an increase in $A P$ concentration in $\mathrm{PM}_{2.5}$ by one unit $\left(81.16 \mu \mathrm{g} / \mathrm{m}^{3}\right)$ was associated with an increase in total weekly hours of SB by 6.24 hours among a large sample $(12,174)$ of university freshmen in China based on a cohort study survey [37]. To our knowledge, this is one of the first studies to investigate the impact of AP on SB by hourly use objectively measured GT3X accelerometers. Because of conflicting results in this emerging area and somewhat preliminary of this finding, additional investigations are necessary to fully explore the effect of AP on SB among different groups.

The impact of AP on individual-level PA and SB at a specific time was different. This study is the first to examine the association between AP on PA and SB at a specific time. Stronger negative associations of AQI and PM 2.5 AP with MVPA, walking steps and energy expenditure in the morning before $8 \mathrm{am}$, at $4 \mathrm{pm}$, at $5 \mathrm{pm}$ and at $7 \mathrm{pm}$ were found. Similarly, stronger positive associations of one hour AQI and one hour $\mathrm{PM}_{2.5}$ on SB at $8 \mathrm{am}, 9 \mathrm{am}, 11 \mathrm{am}$, and $7 \mathrm{pm}$ were found. In this study, all participants were recruited from freshmen. Typically, a substantial proportion of freshmen do not have class before $8 \mathrm{am}$ in the morning, and/or after $4 \mathrm{pm}$. Participants in this study could choose PA or SB behavior according to their own preferences of activities during leisure time. Similar time-specific relationships between built environment and PA were found in the previous studies [40,41]. However, it is interesting that positive associations of $\mathrm{AQI}$ and $\mathrm{PM}_{2.5}$ were found with MVPA, walking steps and energy expenditure in the morning at 10 am and $3 \mathrm{pm}$. Yet, negative associations of $\mathrm{AQI}$ and $\mathrm{PM}_{2.5}$ were found with $\mathrm{SB}$. This could be explained by the fact that there are classes, including physical education class, for a substantial proportion of a freshman students' schedule between 10 am in the morning and $3 \mathrm{pm}$ in the afternoon. Freshmen often engage in PA when traveling to class (e.g. walking or bicycling to or from class) and may perform more exercise in physical education class, regardless of AP. Therefore, $10 \mathrm{am}$ in the morning and $3 \mathrm{pm}$ in the afternoon associations between AP and PA or SB observed in this study are logical. Thus, this study confirms that the impact of AP on PA and SB are different from patterns of in time-specific associations. This finding is closer to the 'true' potential effects of AP on PA and SB.

First, the strengths of this study reside in its objectively measured PA-related behavior and precise reporting of data. Most existing studies on the impact of AP on PA and SB have used subjective methods, allowing for uncontrolled confounding bias due to self-

Page $11 / 15$ 
report and limited frequency of PA data. Second, this is one of the first studies to measure the impact of AP on PA and SB by one hour using objective methods. Third, this is the first study to examine time-specific results on the relationship between AP and objectively measured PA and SB. However, a few limitations to this study should be noted. First, on the one hand, we didn't monitor indoor air pollution and may have bias in this study. A subject's physical activity participation may not vary by air pollution if an individual is active indoors. On the other hand, all freshmen (the subjects of this study) in Tsinghua University live in the same campus, live in the similar dormitories, take similar transportation (e.g., bicycle), and have similar classrooms (6th teaching building in Tsinghua). The subjects sharing similar classrooms, transportation means, and dormitories may partially offset the disruption the influence of indoor air pollution levels. Second, we could not identify the specific types of PA and SB through using accelerometers to assess PA and SB. Third, all participants were recruited by a convenience sampling. Freshman students from one university cannot represent all university students in Beijing, China or nationwide therefore limiting the generalizability of the study's findings. Future studies are warranted to produce more generalized estimates.

\section{Conclusions}

In conclusion, the present study aimed to examine the relationship between AP and hourly behavioral modification to relate objectively-assessed PA and SB among university freshmen in Beijing, China. A negative association between hourly AQI and PM 2.5 and hourly PA in minutes of MVPA, walking steps and energy expenditure among study participants were found. A positive relationship between hourly $\mathrm{AQI}$ and $\mathrm{PM}_{2.5}$ and one-hour $\mathrm{SB}$ modification among study participants was estimated. The impact of $A P$ on individual-level PA and SB at specific time among freshmen was different. This requires policy maker to take action to reduce levels of AP in China. Future studies should consider the replication of the study findings in other groups.

\section{Abbreviations}

BMI: Body mass index; PA: Physical activity; SB: Sedentary behavior; MVPA: Moderate-to-vigorous physical activity; AP: air pollution; AQI: Air quality index; PM2.5: Particulate matter with a diameter less than 2.5 microns.

\section{Declarations}

\section{Acknowledgments}

We are grateful for the support of all the participants in the study. We are grateful to the Beijing Municipal Ecological Environment Bureau provided the hourly $\mathrm{AQI}$ and $\mathrm{PM}_{2.5}$ data and the China Meteorological Administration provided average daytime temperature (C) data in Beijing, China.

\section{Authors' contribution}

HY and MY designed and conceived the study. YW, S P G and J C were involved collected PA and SB data. Y W, JC, P C were involved $\mathrm{AP}$ and ambient temperature data. $\mathrm{HY}$ and $\mathrm{Y} W$ analyzed data. $\mathrm{HY}$ and $\mathrm{M} Y$ wrote the manuscript. All authors read and approved the final manuscript.

\section{Funding}

This study was supported by National Social Science Foundation of China (17CTY020, 16BTY065,19ZDA353) and by The National Key Research and Development Project (2018YFF0300901, 2018YFF0300902) and by the Tsinghua University Initiative Scientific Research Program (20205080066).

\section{Availability of data and materials}

The datasets generated and/or analyzed during the current study are not publicly available due to confidentially reasons, but are available from the corresponding author on reasonable request. 


\section{Ethics approval and consent to participate}

All participants gave informed consent, and the study was approved by the Tsinghua University Institutional Review Board (IRB \#2017DX02_11).

\section{Consent for publication}

All co-authors have agreed in the publication of the submission version of this manuscript.

\section{Competing interests}

The author declares that they have no competing interests.

\section{References}

1. Minos D, Butzlaff I, Demmler KM, Rischke R. Economic Growth, Climate Change, and Obesity. Current Obesity Reports.2016;5:44148.

2. Auchincloss AH, Diez Roux AV, Dvonch JT, Brown PL, Barr RG, Daviglus ML, Goff DC, Kaufman JD, O'Neill MS. Associations between recent exposure to ambient fine particulate matter and blood pressure in the Multi-ethnic Study of Atherosclerosis (MESA). Environ Health Perspect.2008;116:486-91.

3. Billings ME, Gold D, Szpiro A, Aaron CP, Jorgensen N, Gassett A, Leary PJ, Kaufman JD, Redline SR, Jorgensen N, et al. The Association of Ambient Air Pollution with Sleep Apnea: The Multi-Ethnic Study of Atherosclerosis. ANNALS OF THE AMERICAN THORACIC SOCIETY.2019;16:363-70.

4. Brook RD, Rajagopalan S, Pope CA, 3rd, Brook JR, Bhatnagar A, Diez-Roux AV, Holguin F, Hong Y, Luepker RV, Mittleman MA, et al. Particulate matter air pollution and cardiovascular disease: An update to the scientific statement from the American Heart Association. Circulation.2010;121:2331-78.

5. Cakmak S, Dales R, Leech J, Liu L. The influence of air pollution on cardiovascular and pulmonary function and exercise capacity: Canadian Health Measures Survey (CHMS). Environmental Research.2011;111:1309-12.

6. Miller KA, Siscovick DS, Sheppard L, Shepherd K, Sullivan JH, Anderson GL, Kaufman JD. Long-term exposure to air pollution and incidence of cardiovascular events in women. New England Journal of Medicine.2007;356:447-58.

7. Yolton K, Khoury J, Brokamp C, Severs R, Wolfe C, Percy Z, Burkle J, Cecil K, LeMasters G, Ryan P. Associations between Early Life Exposure to Traffic Related Air Pollution and Symptoms of Depression and Anxiety at Age 12 Years. Neurotoxicology and Teratology.2017;61:145-45.

8. Downward GS, Hu W, Rothman N, Reiss B, Tromp P, Wu G, Wei F, Xu J, Seow WJ, Chapman RS. Quartz in ash, and air in a high lung cancer incidence area in China. Environmental Pollution.2017;221:318-25.

9. Ho HC, Wong MS, Yang L, Chan T-C, Bilal M. Influences of socioeconomic vulnerability and intra-urban air pollution exposure on short-term mortality during extreme dust events. Environmental pollution.2018;235:155-62.

10. Wei F, Nie G, Zhou B, Wang L, Ma Y, Peng S, Ou S, Qin J, Li S, Zou R. Association between Chinese cooking oil fumes and sleep quality among a middle-aged Chinese population. Environmental pollution.2017;227:543-51.

11. Department of Health and Human Services: Physical Activity Guidelines for Americans. Department of Health and Human Services; 2008.

12. Lear SA, Hu W, Rangarajan S, Gasevic D, Leong D, Iqbal R, Casanova A, Swaminathan S, Anjana RM, Kumar R, et al. The effect of physical activity on mortality and cardiovascular disease in 130000 people from 17 high-income, middle-income, and lowincome countries: the PURE study. LANCET.2017;390:2643-54.

13. Cradock KA, Olaighin G, Finucane FM, Gainforth HL, Quinlan LR, Ginis KAM. Behaviour change techniques targeting both diet and physical activity in type 2 diabetes: A systematic review and meta-analysis. INTERNATIONAL JOURNAL OF BEHAVIORAL NUTRITION AND PHYSICAL ACTIVITY.2017;14. 
14. Fletcher GF, Landolfo C, Niebauer J, Ozemek C, Arena R, Lavie CJ. Promoting physical activity and exercise: JACC health promotion series. Journal of the American College of Cardiology.2018;72:1622-39.

15. Giles LV, Koehle MS. The health effects of exercising in air pollution. Sports Med.2014;44:223-49.

16. Fisher JE, Ulrik CS, Raaschou-Nielsen O, Loft S, Andersen ZJ, Hertel O, Nieuwenhuijsen MJ, Tjønneland A, Overvad K. Physical Activity, Air Pollution, and the Risk of Asthma and Chronic Obstructive Pulmonary Disease. American Journal of Respiratory and Critical Care Medicine.2016;194:855-65.

17. Z.J A, A dN, M.A M, J G-A, O H, A T, K O, O R-N, M.J N. A study of the combined effects of physical activity and air pollution on mortality in elderly urban residents: The Danish Diet, Cancer, and Health Cohort. Environmental Health Perspectives.2015;123:557-63.

18. Li F, Liu Y, Lu J, Liang L, Harmer P. Ambient air pollution in China poses a multifaceted health threat to outdoor physical activity. J Epidemiol Community Health.2015;69:201-4.

19. Yu H, Yu M, Gordon SP, Zhang R. The association between ambient fine particulate air pollution and physical activity: a cohort study of university students living in Beijing. International Journal of Behavioral Nutrition and Physical Activity.2017;14.

20. Katzmarzyk PT. Physical activity, sedentary behavior, and health: paradigm paralysis or paradigm shift? Diabetes.2010;59:271725.

21. Owen N, Healy GN, Matthews CE, Dunstan DW. Too Much Sitting: The Population Health Science of Sedentary Behavior. Exercise and Sport Sciences Reviews.2010;38:105-13.

22. Owen N, Sparling PB, Healy GN, Dunstan DW, Matthews CE. Sedentary behavior: emerging evidence for a new health risk. Mayo Clin Proc.2010;85:1138-41.

23. de Rezende LFM, Lopes MR, Rey-Lopez JP, Matsudo VKR, Luiz OD. Sedentary Behavior and Health Outcomes: An Overview of Systematic Reviews. Plos One.2014;9.

24. Tremblay MS, LeBlanc AG, Kho ME, Saunders TJ, Larouche R, Colley RC, Goldfield G, Gorber SC. Systematic review of sedentary behaviour and health indicators in school-aged children and youth. International Journal of Behavioral Nutrition and Physical Activity.2011;8.

25. Lavie CJ, Ozemek C, Carbone S, Katzmarzyk PT, Blair SN. Sedentary behavior, exercise, and cardiovascular health. Circulation research.2019;124:799-815.

26. An R, Shen J, Ying B, Tainio M, Andersen ZJ, de Nazelle A. Impact of ambient air pollution on physical activity and sedentary behavior in China: A systematic review. Environmental research.2019:108545.

27. Troiano RP, Berrigan D, Dodd KW, Masse LC, Tilert T, McDowell M. Physical activity in the United States measured by accelerometer. Medicine and science in sports and exercise.2008;40:181.

28. EPA. Air Quality Index (AQI) Basics. 2020. https://airnow.gov/index.cfm?action=aqibasics.aqi Accessed 15, Feburary 2020.

29. Ministry of Ecology and Environment of China C. Air Quality Index(AQI). 2020. http://106.37.208.228:8082/ Accessed 30 Jun,2020 2020.

30. Yu H, An R, Andrade F. Ambient Fine Particulate Matter Air Pollution and Physical Activity: A Longitudinal Study of University Retirees in Beijing, China. American Journal of Health Behavior.2017;41:401-10.

31. An R, Xiang X. Ambient fine particulate matter air pollution and leisure-time physical inactivity among US adults. Public Health.2015;129:1637-44.

32. Wen X-J, Balluz LS, Shire JD, Mokdad AH, Kohl HW, III. Association of Self-Reported Leisure-Time Physical Inactivity with Particulate Matter 2.5 Air Pollution. Journal of Environmental Health.2009;72:40-44.

33. Hu L, Zhu L, Xu Y, Lyu J, Imm K, Yang L. Relationship Between Air Quality and Outdoor Exercise Behavior in China: a Novel Mobile-Based Study. Int J Behav Med.2017;24:520-27.

34. Wen XJ, Balluz LS, Shire JD, Mokdad AH, Kohl HW. Association of self-reported leisure-time physical inactivity with particulate matter 2.5 air pollution. J Environ Health.2009;72:40-4; quiz 45.

35. An R, Yu H. Impact of ambient fine particulate matter air pollution on health behaviors: a longitudinal study of university students in Beijing, China. Public health.2018;159:107-15.

36. Wang G, Li B, Zhang X, Niu C, Li J, Li L, Speakman JR. No seasonal variation in physical activity of Han Chinese living in Beijing. Int J Behav Nutr Phys Act.2017;14:48. 
37. Yu H, Cheng J, Gordon SP, An R, Yu M, Chen X, Yue Q, Qiu J. Impact of Air Pollution on Sedentary Behavior: A Cohort Study of Freshmen at a University in Beijing, China. International journal of environmental research and public health.2018;15:2811.

38. Ma Y, Yuan B, Fan S, Luo Y, Wen X. Association between Air Quality and Sedentary Time in 3270 Chinese Adults: Application of a Novel Technology for Posture Determination. Journal of clinical medicine.2018;7:257.

39. Salmon J, Owen N, Crawford D, Bauman A, Sallis JF. Physical activity and sedentary behavior: a population-based study of barriers, enjoyment, and preference. Health psychology.2003;22:178.

40. Cerin E, Mitáš J, Cain KL, Conway TL, Adams MA, Schofield G, Sarmiento OL, Reis RS, Schipperijn J, Davey R. Do associations between objectively-assessed physical activity and neighbourhood environment attributes vary by time of the day and day of the week? IPEN adult study. International Journal of Behavioral Nutrition and Physical Activity.2017;14:34.

41. Arvidsson D, Eriksson U, Lönn SL, Sundquist K. Neighborhood walkability, income, and hour-by-hour physical activity patterns. Medicine and science in sports and exercise.2013;45:698-705.

\section{Figures}

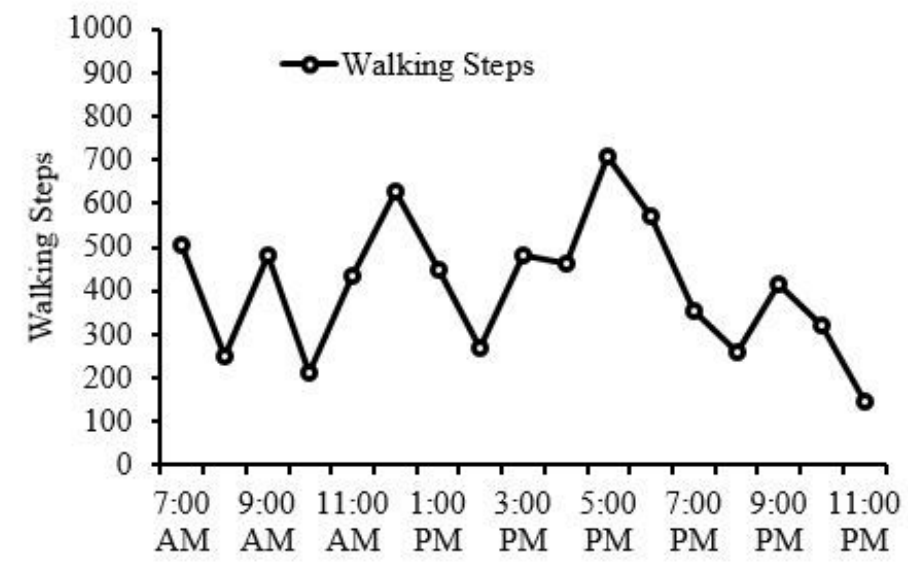

\section{Figure 1}

Trend for walking steps in specific time in one day among the freshman. 